\title{
Disfunções cognitivas em pacientes com hipotireoidismo subclínico
}

\author{
Cognitive dysfunction in patients with subclinical hypothyroidism
}

Robertta Soares Miranda Fernandes', Nathália Bueno Alvarenga ${ }^{2}$, Tamara Inácio da Silva' , Felipe Filardi da Rocha ${ }^{1,2,3}$

\begin{abstract}
RESUMO
Objetivo: Avaliar a presença de disfunções cognitivas em pacientes com hipotireoidismo subclínico (HS). Sujeitos e métodos: Estudo seccional comparando os achados neuropsicológicos de 89 pacientes com HS e 178 indivíduos eutireoidianos. Para avaliação neuropsicológica, foram utilizados: Teste de Performance Contínua (CPT-II), lowa Gambling Task, Teste de Stroop, Teste de Wisconsin (WCST), Teste da Figura Complexa de Rey-Osterreich, Teste de Fluência Verbal (categorias semântica e fonológica) e Teste de Aprendizagem Auditivo Verbal de Rey. Resultados: Os pacientes mostraram pior desempenho na flexibilidade cognitiva (WCST) e na capacidade de manter atenção sustentada (erros por omissão no CPT-II). Conclusões: Esses déficits podem causar prejuízos na vida diária do paciente, constituindo possíveis indicações de tratamento. Arq Bras Endocrinol Metab. 2011;55(3):224-8

Descritores

Hipotireoidismo subclínico; transtornos cognitivos; neuropsicologia; atenção
\end{abstract}

\section{ABSTRACT}

Correspondência para: Felipe Filardi da Rocha Rua Sapucaia, 83

35460-000 - Brumadinho, MG, Brasil fil_bh@yahoo.com.br

Recebido em 11/Jan/2011 Aceito em 18/Fev/2011

Objective: Evaluate neuropsychological changes in patients with subclinical hypothyroidism (SH). Subjects and methods: Cross-sectional study comparing the results of the neuropsychological evaluation of $89 \mathrm{SH}$ patients and 178 individuals without thyroid disease. The participants underwent the following neuropsychological assessment: Conner's Continuous Performance Test (CPT-II), lowa Gambling Task, Stroop Test, Wisconsin Card Sorting Test (WCST), Verbal Fluency Test (semantic and phonologic categories) and Rey Auditory Verbal Learning Test. Results: Among the neuropsychological tests, patients showed worse performance only in cognitive flexibility (WCST) and the ability to maintain sustained attention (omission errors on the CPT-II). Conclusions: These losses can cause detriments in the daily lives of patients, constituting potential treatment indications. Arq Bras Endocrinol Metab. 2011;55(3):224-8

\section{Keywords}

Subclinical hypothyroidism; cognition disorders; neuropsychology; attention

\section{INTRODUÇÃO}

$\mathrm{O}$ hipotireoidismo subclínico (HS) é uma condição endócrina relativamente comum, acometendo, aproximadamente, entre $1 \%$ e $15 \%$ da população (1-3). Enquanto alguns pesquisadores relatam que os pacientes podem evoluir para hipotireoidismo clínico com progressão diretamente proporcional aos níveis de TSH, outra parcela dos pacientes não apresentará evolução do quadro, requerendo apenas observação clínica. Dessa forma, o tratamento e a abordagem são questões de grande discussão entre clínicos e pesquisadores $(1,2)$.
Apesar de não haver consenso em quais momentos o tratamento farmacológico com a levotiroxina deve ser iniciado, sugere-se seu uso na presença de sintomas clínicos de hipotireoidismo, na presença de dislipidemias e níveis constantes e elevados de anticorpos, principalmente os anticorpos antiperoxidase (1-4).

Um grupo de alterações observadas em quadros de hipotireoidismo clínico são as alterações neurocognitivas, com diversas disfunções, como, por exemplo, dificuldade de manter atenção sustentada, lentificação psicomotora e prejuízos da memória de fixação. Esses sintomas decorrem da importante ação da triiodotironi- 
na no metabolismo celular, principalmente no sistema nervoso central (3).

Estudos têm sugerido que pacientes com HS poderiam também manifestar alterações neuropsicológicas $(4,5)$. Essas alterações cursariam consequentemente com prejuízos para o paciente mesmo que, muitas vezes, sejam percebidos apenas com testes específicos $(5,6)$.

A provável presença desses fatores é pouco explorada na literatura científica. Sua presença, sendo reversível com o tratamento farmacológico, poderia ser uma indicação formal para o início deste (2).

Nosso objetivo é avaliar, de forma extensa, diversas funções neurocognitivas de pacientes com HS, comparando com grupo controle constituído de indivíduos hígidos. Trabalhamos com a hipótese de que os pacientes devem apresentar alterações neuropsicológicas e essas talvez estejam associadas a fatores clínicos e/ou laboratoriais.

\section{SUJEITOS E MÉTODOS}

Trata-se de estudo caso-controle realizado na Universidade Federal de Minas Gerais, Belo Horizonte, Brasil, pelo serviço de psiquiatria e psicologia. Durante o período de três anos, foram avaliados, de forma consecutiva, todos os pacientes com diagnóstico de HS confirmado por duas medidas laboratoriais de TSH e T4 livre. É importante ressaltar que este estudo foi aprovado pela Comissão de Ética da Instituição de Pesquisa onde o estudo foi realizado.

\section{Avaliação dos pacientes}

O paciente passava por avaliação clínica e psiquiátrica completa. Os critérios de exclusão foram: presença de sintomas clínicos compatíveis com hipotireoidismo (ganho de peso significativo nos últimos dois meses, queda importante do cabelo e apatia e/ou sensação de cansaço nos dois últimos meses), transtornos psiquiátricos (avaliados pelo MINIPLUS, escala padronizada e validada para o português), uso de fármacos que sabidamente alteram a cognição (antiepilépticos, psicofármacos e medicações sedativas, como, por exemplo, difenidramina), outras doenças endócrinas, cardiovasculares e neurológicas, história de traumas cranioencefálicos pregressos e pacientes abaixo do percentil 25 nas Escalas Progressivas de Raven.

Em seguida, o paciente foi esclarecido a respeito dos objetivos da pesquisa e o Termo de Esclarecimento Livre Esclarecido foi assinado. Com a aceitação, dados sociodemográficos (idade, gênero, escolaridade) e labo- ratoriais (TSH, T4 livre) foram recolhidos e/ou solicitados e aplicamos as seguintes escalas e bateria de exames:

- Escala de Depressão de Beck e Escala de Ansiedade de Beck: apesar de a presença de sintomas psiquiátricos serem fatores de exclusão, a simples presença se sintomas depressivos e/ou ansiosos pode ser fator de confusão na avaliação neuropsicológica;

- Escalas Progressivas de Raven: para mensurar a inteligência do paciente, fator diretamente associado à adequada realização dos testes e preenchimento das escalas.

- Bateria de testes neuropsicológicos para avaliar atenção, memória, tomada de decisão, fluência verbal e aprendizagem verbal: Teste de Performance Contínua (CPT-II), Iowa Gambling Task (IGT), Teste de Stroop, Teste de Wisconsin, Teste da Figura Complexa de Rey-Osterreich, Teste de Fluência Verbal (categorias semântica e fonológica) e Teste de Aprendizagem Auditivo Verbal de Rey (RAVLT).

Os testes foram realizados por neuropsicólogo experiente, sempre na mesma hora do dia (nove horas da manhã), em três sessões de duas horas cada.

\section{Seleção e avaliação dos controles}

Os controles foram selecionados entre estudantes, funcionários e voluntários convocados para o estudo. Foram pareados por sexo, idade e escolaridade na proporção de dois controles para cada paciente, totalizando 178 controles e 89 pacientes. Os controles foram submetidos a todas as etapas pelas quais os pacientes passaram. Os critérios de exclusão foram: presença de transtornos psiquiátricos (avaliados pelo MINIPLUS), uso de fármacos que sabidamente alteram a cognição (antiepilépticos, psicofármacos e medicações sedativas, como, por exemplo, difenidramina), doenças endócrinas, cardiovasculares e neurológicas, história de traumas cranioencefálicos pregressos e pacientes abaixo do percentil 25 na Escalas Progressivas de Raven. Além disso, qualquer história de doença tireoidiana na família também serviu como critério de exclusão.

\section{Análise estatística}

Toda a análise estatística foi realizada com o software SPSS v. 11.5. Controles e casos foram comparados com teste de Mann-Whitney (varáveis contínuas) e teste qui-quadrado (variáveis categóricas). Teste de correlação de Spearman foi realizado para avaliar a influência dos exames laboratoriais (TSH, T4 livre), idade, e dos sintomas ansiosos e depressivos no desempenho neuropsicológico de controles e pacientes. O nível de significância foi de 5\%. 


\section{RESULTADOS}

Casos e controles não apresentaram diferenças entre gênero, idade, inteligência, escolaridade, sintomas depressivos e ansiosos. No entanto, como já esperado pela própria proposta do estudo, os níveis de TSH foram bem maiores entre os pacientes, mas com níveis normais de T4 livre (Tabela 1).

Tabela 1. Diferenças clínicas, laboratoriais e neuropsicológicas entre casos e controles

\begin{tabular}{|c|c|c|c|}
\hline Fatores clínicos e demográficos & $\begin{array}{c}\text { Pacientes } \\
(\mathbf{n}=\mathbf{8 9})\end{array}$ & $\begin{array}{l}\text { Controles } \\
(\mathrm{n}=178) \\
\end{array}$ & Valor $\mathbf{p}$ \\
\hline Idade, mês $\pm \mathrm{DP}$, anos & $35,40 \pm 14,12$ & $33,34 \pm 13,22$ & $P=0,24$ \\
\hline Gênero masculino (\%) & $17,97 \%(n=16)$ & $17,97 \%(n=32)$ & $P>0,05$ \\
\hline Anos de educação formal $\pm \mathrm{DP}$, anos & $09,56 \pm 4,87$ & $09,26 \pm 5,28$ & $P=0,65$ \\
\hline Inteligência & $42,48 \pm 6,75$ & $42,12 \pm 7,88$ & $P=0,71$ \\
\hline Escore no BDI & $8,89 \pm 4,75$ & $9,01 \pm 6,22$ & $P=0,86$ \\
\hline Escore no BAl & $11,39 \pm 5,09$ & $10,21 \pm 7,44$ & $P=0,12$ \\
\hline TSH (mUI/L) & $9,53 \pm 4,02$ & $1,89 \pm 1,75$ & $P<0,05$ \\
\hline T4 livre (ng/dL) & $1,34 \pm 0,22$ & $1,29 \pm 0,24$ & $P=0,10$ \\
\hline \multicolumn{4}{|l|}{ Iowa Gambling Task } \\
\hline IGT - Escore total & $5,98 \pm 10,34$ & $5,64 \pm 12,73$ & $P=0,81$ \\
\hline IGT $-[(C+D)-(A=B)]$ Primeiro bloco de 50 cartas & $1,94 \pm 6,84$ & $1,34 \pm 8,48$ & $P=0,53$ \\
\hline IGT $-[(C+D)-(A=B)]$ Segundo bloco de 50 cartas & $4,69 \pm 6,66$ & $4,38 \pm 9,41$ & $P=0,75$ \\
\hline \multicolumn{4}{|l|}{ Teste de Stroop } \\
\hline Stroop 1 (nome da cor) & $14,43 \pm 1,32$ & $14,76 \pm 2,32$ & $P=0,14$ \\
\hline Stroop 2 (cor da palavra) & $15,88 \pm 1,73$ & $15,76 \pm 1,84$ & $P=0,60$ \\
\hline Stroop 3 (interferência cor/palavra) & $19,45 \pm 1,90$ & $19,70 \pm 1,82$ & $P=0,30$ \\
\hline Stroop 1 (erros do nome da cor) & $0,02 \pm 0,31$ & $0,04 \pm 0,46$ & $P=0,67$ \\
\hline Stroop 2 (erros da cor da palavra) & $0,01 \pm 0,01$ & $0,01 \pm 0,11$ & $P=1,00$ \\
\hline Stroop 3 (erros da interferência cor/palavra) & $0,02 \pm 0,45$ & $0,01 \pm 0,64$ & $P=0,88$ \\
\hline \multicolumn{4}{|l|}{ Teste de Performance Contínua } \\
\hline Erros de omissão & $2,53 \pm 3,29$ & $17,42 \pm 21,59$ & $P<0,05$ \\
\hline Erros de comissão & $11,07 \pm 8,26$ & $9,98 \pm 7,34$ & $P=0,27$ \\
\hline \multicolumn{4}{|l|}{ Teste de WCST } \\
\hline Total de acertos & $40,76 \pm 7,35$ & $27,50 \pm 9,63$ & $P<0,05$ \\
\hline Erros perseverativos & $7,74 \pm 5,03$ & $16,83 \pm 8,51$ & $P<0,05$ \\
\hline Categorias completas & $2,87 \pm 1,20$ & $1,14 \pm 1,06$ & $P<0,05$ \\
\hline Falhas em se manter na categoria & $0,23 \pm 0,65$ & $0,69 \pm 0,82$ & $P<0,05$ \\
\hline \multicolumn{4}{|l|}{ Figura complexa de Rey } \\
\hline Total de pontos & $32,63 \pm 5,27$ & $32,01 \pm 8,00$ & $P=0,45$ \\
\hline Reconstituição após três minutos & $27,74 \pm 6,35$ & $25,98 \pm 8,92$ & $P=0,06$ \\
\hline \multicolumn{4}{|l|}{ Fluência verbal } \\
\hline Fluência verbal fonológica - F & $13,79 \pm 3,28$ & $14,01 \pm 4,21$ & $P=0,63$ \\
\hline Fluência verbal fonológica - A & $9,04 \pm 4,12$ & $9,37 \pm 3,06$ & $P=0,50$ \\
\hline Fluência verbal fonológica - S & $13,59 \pm 3,73$ & $13,03 \pm 3,54$ & $P=0,23$ \\
\hline Fluência verbal semântica (animais) & $19,42 \pm 3,19$ & $18,95 \pm 4,98$ & $P=0,35$ \\
\hline Fluência verbal fonológica F - erros perseverativos & $0,91 \pm 1,48$ & $0,95 \pm 1,27$ & $P=0,81$ \\
\hline Fluência verbal fonológica A - erros perseverativos & $0,70 \pm 0,83$ & $0,82 \pm 1,22$ & $P=0,34$ \\
\hline Fluência verbal fonológica S - erros perseverativos & $0,99 \pm 0,71$ & $0,95 \pm 0,80$ & $P=0,68$ \\
\hline Fluência verbal semântica de animais - erros perseverativos & $1,43 \pm 1,24$ & $1,21 \pm 2,98$ & $P=0,39$ \\
\hline \multicolumn{4}{|l|}{ RAVLT } \\
\hline A1-A5 & $51,76 \pm 7,80$ & $50,91 \pm 9,74$ & $P=0,44$ \\
\hline Interferência proativa & $0,82 \pm 0,19$ & $0,80 \pm 0,21$ & $P=0,44$ \\
\hline Interferência retroativa & $0,79 \pm 0,23$ & $0,81 \pm 0,20$ & $P=0,46$ \\
\hline Palavras esquecidas & $0,91 \pm 0,22$ & $0,90 \pm 0,23$ & $P=0,73$ \\
\hline Memória de reconhecimento & $12,47 \pm 2,98$ & $12,22 \pm 3,84$ & $P=0,55$ \\
\hline
\end{tabular}


Nos testes neuropsicológicos, os pacientes mostraram desempenho pior na flexibilidade cognitiva (WCST) e na capacidade de manter atenção sustentada (erros por omissão no CPT-II). Nenhum outro teste da bateria utilizada mostrou alteração entre casos e controles.

Avaliamos possíveis correlações entre idade, inteligência, sintomas ansiosos, sintomas depressivos, níveis de TSH e níveis de T4 livre com os diversos testes aplicados.

Os níveis de TSH mostraram uma forte correlação positiva com os erros de omissão no CPT-II (Rho = $0,91 ; \mathrm{p}<0,01)$. Todos os outros resultados mostraram fracas correlações $(-0,20<$ Rho $<0,19)$ sem níveis de significância $(\mathrm{p}>0,05)$.

\section{DISCUSSÃO}

Observamos que pacientes com HS apresentaram prejuízos cognitivos em dois testes neuropsicológicos. Mais especificamente no WCST (flexibilidade cognitiva) e no CPT-II (dificuldade de sustentar atenção), testes associados a funções executivas da região dorsolateral do córtex pré-frontal $(6,7)$.

Esses prejuízos, apesar de poderem passar despercebidos na prática clínica, podem estar associados com problemas no trabalho, nos relacionamentos sociais, acidentes de trânsito, entre outros (2,4-6).

Poucos são os estudos que avaliaram déficits cognitivos nesses pacientes, apesar do provável comprometimento de funções cerebrais superiores, sugerido pela frequente presença de sintomas neuropsiquiátricos, como, por exemplo, sintomas depressivos $(8,9)$. Reforçando essa ideia, uma das plausíveis indicações de tratamento do HS é a presença concomitante de sintomas psiquiátricos $(2,8,9)$.

Os estudos que avaliam possíveis disfunções cognitivas no HS são inconclusivos, sem alterações específicas determinadas e alguns achados negativos.

Baldini e cols. (5) observaram prejuízo na memória lógica avaliada com Wechsler Memory Scale. Bono e cols. (10) evidenciaram, em mulheres com HS, dificuldades de sustentar a atenção que pioravam com o aumento da idade. Contudo, Jorde e cols. (11) não observaram diferenças entre casos e controles ao usarem uma bateria de testes neuropsicológicos. Neste mesmo ano, um estudo com ressonância magnética funcional demonstrou que pacientes com HS apresentavam prejuízos na memória de trabalho, com evidência de comprometimento das seguintes regiões cerebrais: giros frontais inferior e médio, córtex pré-frontal dorsolate- ral, área pré-motora, área motora suplementar, cíngulo anterior e regiões do córtex parietal (12).

Mais recentemente, Almeida e cols. (4) estudaram 65 pacientes com HS e 31 controles. Os testes utilizados avaliaram cognição verbal (Wechsler Adult Intelligence Scale - subitem que avalia vocabulário), capacidade de aprendizagem (Buschke's Selective Reminding Procedure) e teste de memória visual (Rey-Osterreich complex figure test). Apesar de nenhuma diferença ter sido observada, as habilidades avaliadas diferem das funções cognitivas que observamos prejuízo em nosso estudo. Corroborando esse trabalho, não observamos também alterações na fluência verbal, no mesmo teste de memória visual e na capacidade de aprendizagem.

Em um recente estudo, idosos com HS apresentaram pior desempenho no Miniexame do Estado Mental (MEEM) (13). No entanto, algumas críticas podem ser feitas. Inicialmente, pessoas idosas podem ser mais suscetíveis a alterações neuroendócrinas devido à redução fisiológica da sua reserva cerebral. Além disso, o MEEM apresenta pouca especificidade, não reforçando de forma mais detalhada os domínios cognitivos a que se propõem avaliar em cada item.

Interessantemente, nossos achados corroboram dados pregressos de correlação positiva entre os níveis de TSH e o prejuízo cognitivo $(1,14)$. Algumas questões são levantadas a partir dessas observações. O provável funcionamento neuronal deveria estar associado aos níveis hormonais tireoidianos que, no HS, estão normais, resultando, assim, em achados negativos $(1,2)$. Dessa forma, deve-se pensar na possibilidade de existirem outros marcadores laboratoriais mais específicos que, de forma direta ou indireta, estariam associados aos níveis de TSH. Essa relação, entre níveis de TSH e disfunção cognitiva, foi recentemente avaliada em uma coorte com 178 recém-nascidos, em que níveis de TSH no cordão umbilical apresentaram uma correlação positiva com disfunções executivas futuras (15). Alternativamente, níveis de TSH podem exercer feed-back negativo em vias neuroendócrinas associadas ao desempenho cognitivo, ainda não totalmente elucidadas ( 1 $3,5,11,16)$.

O presente trabalho apresenta a vantagem de estudar funções executivas associadas às três principais regiões do córtex pré-frontal: regiões dorsolateral, cíngulo anterior e orbitofrontal. As alterações observadas dizem respeito à região dorsolateral, fato também observado em outros estudos, aparentando ser a região associada às principais disfunções observadas no HS. Além dis- 
so, nossa amostra foi extremamente bem caracterizada, com rígidos critérios de inclusão e exclusão, além de pareamento entre casos e controles no que diz respeito a gênero, idade, anos de educação formal e inteligência. Esses fatores são importantes, pois podem alterar testes neuropsicológicos. Além disso, mesmo excluindo transtornos psiquiátricos, tivemos o cuidado de avaliar a presença de sintomas ansiosos e depressivos, o que poderia, também, alterar nossos resultados.

No entanto, não podemos generalizar esses achados. São necessários trabalhos com números maiores de pacientes para confirmar e detalhar de forma mais sucinta essas alterações neurocognitivas, além de avaliar outras variáveis, como, por exemplo, anticorpos antitireoidianos (1-4).

Fato que também requer maiores estudos é a provável reversibilidade dessas alterações com o uso da levotiroxina. Diversos trabalhos têm mostrado melhora do desempenho cognitivo com o tratamento, porém sem consenso definido na literatura $(10,11,14,17,18)$. Contudo, caso seja evidenciada a potencialidade do tratamento nesses casos, a subsequente melhora da qualidade de vida deve ser analisada para se avaliar uma indicação formal do uso da levotiroxina em pacientes com HS.

Agradecimentos: Nosso agradecimento à Fundação de Amparo à Pesquisa do Estado de Minas Gerais (Fapemig), que financiou este estudo.

Declaração: os autores declaram não haver conflitos de interesse científico neste estudo.

\section{REFERÊNCIAS}

1. Biondi B, Cooper DS. The clinical significance of subclinical thyroid dysfunction. Endocr Rev. 2008;29(1):76-131. Epub 2007 Nov 8.

2. Davis JD, Tremont G. Neuropsychiatric aspects of hypothyroidism and treatment reversibility. Minerva Endocrinol. 2007;32(1):49-65.

3. Razvi S, Weaver JU, Pearce SH. Subclinical thyroid disorders: significance and clinical impact. J Clin Pathol. 2010;63(5):379-86.
4. Almeida C, Vaisman M, Costa AJ, Reis FA, Reuters V, Teixeira P, et al. Are neuropsychological changes relevant in subclinical hypothyroidism? Arq Bras Endocrinol Metabol. 2007;51(4):606-11.

5. Baldini IM, Vita A, Massimo C, Amodei V, Carrisi M, Bravin S, et al. Psychopathological and cognitive features in subclinical hypothyroidism. Prog Neuropsychopharmacol Biol Psychiatry. 1997;21(6):925-35.

6. da Rocha FF, Malloy-Diniz L, Lage NV, Romano-Silva MA, de Marco LA, Correa H. Decision-making impairment is related to serotonin transporter promoter polymorphism in a sample of patients with obsessive-compulsive disorder. Behav Brain Res. 2008;195(1):159-63.

7. da Rocha FF, Malloy-Diniz L, de Sousa KC, Prais HA, Correa H, Teixeira AL. Borderline personality features possibly related to cingulate and orbitofrontal cortices dysfunction due to schizencephaly. Clin Neurol Neurosurg. 2008;110(4):396-9.

8. Almeida C, Brasil MA, Costa AJ, Reis FA, Reuters V, Teixeira P, et al. Subclinical hypothyroidism: psychiatric disorders and symptoms. Rev Bras Psiquiatr. 2007;29(2):157-9.

9. Demartini B, Masu A, Scarone S, Pontiroli AE, Gambini O. Prevalence of depression in patients affected by subclinical hypothyroidism. Panminerva Med. 2010;52(4):277-82.

10. Bono G, Fancellu R, Blandini F, Santoro G, Mauri M. Cognitive and affective status in mild hypothyroidism and interaction with L-thyroxine treatment. Acta Neurol Scand. 2004;110:59-66.

11. Jorde R, Waterloo K, Storhaug H, Nyrnes A, Sundsfjord J, Jenssen TG. Neuropsychological function and symptoms in subjects with subclinical hypothyroidism and the effect of thyroxine treatment. J Clin Endocrinol Metab. 2006;91(1):145-53.

12. Zhu DF, Wang ZX, Zhang DR, Pan ZL, He S, Hu XP, et al. fMRI revealed neural substrate for reversible working memory dysfunction in subclinical hypothyroidism. Brain. 2006;129(11):2923-30.

13. Ceresini G, Lauretani F, Maggio M, Ceda GP, Morganti S, Usberti $E$, et al. Thyroid function abnormalities and cognitive impairment in elderly people: results of the Invecchiare in Chianti study. J Am Geriatr Soc. 2009;57(1):89-93.

14. Jensovský J, Spacková N, Hejduková B, Růzicka E. Effect of normalization of an isolated increase in TSH on the neuropsychological profile of patients. Cas Lek Cesk. 2000;139(10):313-6.

15. Freire $C$, Ramos $R$, Amaya $E$, Fernández MF, Santiago-Fernández $P$, Lopez-Espinosa MJ, et al. Newborn TSH concentration and its association with cognitive development in healthy boys. Eur $\mathrm{J}$ Endocrinol. 2010;163(6):901-9. Epub 2010 Sep 9.

16. Rosario PW, Xavier ACM, Calsolari MR. TSH reference values for adult Brazilian population. Arq Bras Endocrinol Metab. 2010;54:603-6.

17. Santoro G, Mauri M. Cognitive and affective status in mild hypothyroidism and interaction with L-thyroxine treatment. Acta Neurol Scand. 2004;110:59-66.

18. Davis JD, Stern RA, Flashman LA. Cognitive and neuropsychiatric aspects of subclinical hypothyroidism: significance in the elderly. Curr Psychiatry Rep. 2003;5(5):384-90. 